\title{
Liquidez de los mercados accionarios latinoamericanos y su impacto en el crecimiento económico per-cápita
}

Recibido: marzo, 26 de 2019 - Aprobado: junio, 28 de 2019

Doi: http://dx.doi.org/10.12804/revistas.urosario.edu.co/economia/a.8144

\author{
Freddy Benjamin Naula Sigua* \\ Jorge Arturo Campoverde Campoverde ${ }^{\dagger}$ \\ Pedro Fabian Mora Pacheco \\ Diego Mauricio Loyola Ochoa§
}

\section{Resumen}

El presente artículo aporta evidencia empírica respecto a la influencia que tiene la liquidez del mercado accionario en el crecimiento económico, para una muestra de 11 países latinoamericanos. Con ésta finalidad, se utilizaron datos de panel, estimados por el método de Momentos Generalizados (GMM por sus siglas en inglés), además de variantes de dicho modelo. A pesar de que uno de los indicadores de liquidez, específicamente el Turnover, presenta un signo negativo, lo cual es contrario a lo típicamente esperado, a nivel general es posible concluir que la liquidez sí influye en el crecimiento económico. Además, existen países que pueden ser considerados atípicos; por eso se realizó el análisis con y sin ellos, obteniendo en ambos casos que se mantenía el mismo resultado. El estudio se corresponde de forma parcial con la literatura existente.

Palabras clave: liquidez, crecimiento económico, mercado accionario, Latinoamérica. Clasificación JEL: C23, E66, G10, 016

\footnotetext{
* Universidad de Cuenca. Correo electrónico: freddybenja@gmail.com

† Universidad de Cuenca. Correo electrónico: jorge.campoverde@ucuenca.edu.ec

‡ Universidad de Cuenca. Correo electrónico: pedro.mora@ucuenca.edu.ec

$\S$ Universidad de Cuenca. Correo electrónico: diego.loyola@ucuenca.edu.ec
}

Para citar este artículo: Naula, F. B. (2019). Liquidez de los mercados accionarios latinoamericanos y su impacto en el crecimiento económico per cápita. Revista de Economía del Rosario 22(2), 371-392. Doi: http://dx.doi.org/10.12804/revistas.urosario.edu.co/economia/a.8144 


\title{
Latin American Stock Markets Liquidity and their Impact in the Per Capita Economic Growth
}

\begin{abstract}
This article provides empirical evidence regarding the influence of the liquidity of the stock market for economic growth, for a sample of 11 Latin American countries. For this purpose, researchers used panel data estimated by the generalized moment method (GMM), as well as variants of the model. The liquidity indicator: turn over, presented a negative sign, which is contrary to what is typically expected. Broadly, it is possible to conclude that liquidity influences economic growth. In addition, there are countries that can be considered atypical; because of this, the analysis was carried out with and without them. However, the results are maintained. The study corresponds partially to the existing literature.
\end{abstract}

Keywords: Liquidity, economic growth, stock market, Latin America.

JEL Classification: C23, E66, G10, 016.

\section{Liquidez dos mercados acionários Latino-americanos e seu impacto no crescimento econômico per capita}

\begin{abstract}
Resumo
O presente artigo aporta evidência empírica respeito à influência que tem a liquidez do mercado acionário no crescimento econômico, para uma amostra de 11 países latinoamericanos. Com esta finalidade utilizaram-se Dados de Painel, estimando pelo método de Momentos Generalizados (GMM por suas siglas em inglês), para além de variantes de dito modelo. Apesar que um dos indicadores de liquidez, especificamente Turnover, apresenta um signo negativo, o que é contrário ao tipicamente esperado; no nível geral é possível concluir que a liquidez sim influi no crescimento econômico. Para além disso, existem países que podem ser considerados atípicos, devido a isto realizou-se a análise com e sem eles; no entanto, os resultados em essência se mantêm. O estudo se corresponde de forma parcial com a literatura existente.
\end{abstract}

Palavras-chave: liquidez, crescimento econômico, mercado acionário, Latino-América. Classificação JEL: C23, E66, G10, 016. 


\section{Introducción}

La calidad y cantidad de la inversión comprometida por una economía es crucial: las teorías económicas heterodoxas y dominantes resaltan la formación de capital como uno de los principales factores, si no el más importante, en el proceso de desarrollo (Lloyd, 1977). Partiendo desde este punto, parece obvio suponer que los mercados de capitales, mientras más desarrollados, facilitan mejor el flujo de recursos hacia las actividades que requieren financiamiento, lo que a criterio de Levine y Zervos (1998b) se traduciría en una mayor liquidez. En este contexto, King et al. (1993) menciona que el buen funcionamiento de los intermediarios y mercados financieros mejora los costos de transacción e información, lo cual incentivaría la asignación eficiente de recursos, repercutiendo en un crecimiento económico de largo plazo más acelerado.

El sustento empírico que respalda los conceptos anteriores no se ha hecho esperar. Por ejemplo, Lu (2013) encuentra que la eficiencia financiera contribuye al crecimiento económico de largo plazo. A su vez Beck y Levine (2004) evidencia que el desarrollo de los mercados accionarios y de los bancos tiene un impacto positivo y significativo en el crecimiento económico. Atje y Jovanovic (1993) descubren un gran efecto de los mercados accionarios en el desarrollo subsecuente de una economía. En esta misma línea, existen estudios adicionales que fortalecen lo dicho (Cooray, 2010; Al Nasser, 2015; Durusuciftci et al., 2017; Ruiz, 2018). En definitiva, y como menciona Demirgui-kunt y Levine (1996), los mercados accionarios pueden influir en el crecimiento económico, a través de cambios en las políticas de los gobiernos corporativos, facilitando la salida de los inversionistas mediante IPO's, proveyendo fuentes de financiamiento alternativas a los bancos y/o permitiendo la evaluación de las empresas al existir un mayor flujo de información (Francis et al., 2015). Centrándonos en el papel desempeñado por el mercado accionario y, en específico, en la liquidez de éste, resulta cuando menos interesante que a dicha medida también se le atribuya una connotación negativa (Bencivenga et al., 1996). En este contexto, existen tres puntos de vista que explicarían la mencionada situación. Primero, un incremento de la liquidez del mercado podría reducir las tasas de ahorros a través de los efectos ingreso y sustitución. En caso de que las tasas de ahorro cayeran lo suficiente y si hubiera una externalidad vinculada a la acumulación de capital, una gran liquidez de mercado podría lentificar el crecimiento económico. Segundo, para reducir la incertidumbre asociada con la inversión, una gran liquidez del mercado accionario puede reducir las tasas de ahorro a causa de los efectos ambiguos de la incertidumbre en los ahorros; así, menos incertidumbre también disminuye la demanda por ahorros precautorios. Por lo tanto, el impacto último de 
una baja incertidumbre en los ahorros, producidos por una alta liquidez del mercado accionario es incierto. Tercero, la liquidez del mercado accionario podría provocar efectos adversos en los gobiernos corporativos; mercados demasiado líquidos pueden dar paso a la miopía del inversor, debido a que en un mercado altamente líquido un inversor insatisfecho venderá rápidamente sus activos, lo cual evidenciaría una reducción en el compromiso de aquel para con el control y monitoreo del desempeño corporativo de las empresas en general. Es decir, una liquidez de mercado demasiado grande podría eventualmente frenar el crecimiento económico (Demirgui-kunt \& Levine, 1996).

Lo anterior tiene su respaldo en Harris (1997), para quien la relación entre el nivel de la actividad del mercado de acciones y el crecimiento económico es débil. Esta situación es desconcertante, más aún si se considera lo inicialmente mencionado; además, autores como Levine y Zervos (1998b), y Fufa y Kim (2018) demuestran que la liquidez del mercado accionario influye positivamente en el crecimiento económico. Por lo tanto, la finalidad y contribución principal de la presente investigación cae no tanto en el campo de un avance metodológico ${ }^{1}$ sino en el de la evidencia empírica, a nivel de Latinoamérica.

Ciertamente existen algunos estudios relacionados, como los de Terceño y Guercio (2011) y Aali-Bujari et al. (2017), quienes parecerían enfocarse tan solo en la capitalización bursátil, pero descartan de plano la liquidez. El primero limita su análisis al uso de una correlación, mientras que el segundo utiliza datos de panel estimando por GMM (Generalized Method of Moments). Por su parte, Al Nasser (2015) adopta el mismo enfoque que la presente investigación; sin embargo, el método de estimación difiere del de nuestra investigación. De lo anterior se puede inferir que el impacto de la liquidez de los distintos mercados accionarios en el crecimiento económico de los países latinoamericanos, con datos de panel estimados por GMM, aún no se ha llevado cabo.

En términos generales, el presente artículo determina la influencia de la liquidez del mercado accionario con respecto al crecimiento económico, para lo cual utiliza dos medidas: Turnover y Valued Traded, los cuales se consideran proxies de la liquidez. El estudio, además, se vale de datos de panel, estimados por GMM. Este método es utilizado principalmente por aspectos de endogeneidad entre el crecimiento económico y el desarrollo de los mercados accionarios. Los países considerados son en el estudio son Argentina, Barbados, Bermuda, Brasil, Chile, Colombia, Costa Rica, Jamaica, México, Panamá y Perú. Se prescinde del resto de países debido a la ausencia de da-

1 Entendido como un avance estrictamente econométrico. 
tos de sus mercados accionarios. Por otra parte, como Barbados, Bermudas y Jamaica pueden ser considerados como atípicos (outliers), las estimaciones se la realizaron con y sin ellos; los resultados no varían sustancialmente al realizar esta segregación.

En lo que sigue del trabajo se describen, en sus respectivas secciones, una breve revisión bibliográfica, el aspecto metodológico, la data, los resultados, una discusión y las conclusiones.

\section{Revisión bibliográfica}

Si bien el concepto se le atribuye a Schumpeter (1911), dentro de la literatura concerniente al vínculo del desarrollo financiero con el crecimiento económico, Goldsmith (1969) es considerado como el pionero en demostrar que entre dichos aspectos existe una relación positiva, aun cuando también se nombran los trabajos de McKinnon (1973) y Shaw (1973). Posteriormente, varios trabajos adicionales se han publicado, entre ellos el de Levine (1991), quien plantea un modelo en el cual los mercados accionarios aceleran el crecimiento al facilitar el comercio de la propiedad de una firma sin interrumpir el proceso productivo interno, permitiendo, además, la diversificación del portafolio. Levine (1991) concluye, entre otras cosas, que la liquidez adquiere una connotación negativa en un contexto en el cual no se cuenta con mercados accionarios, ello debido en esencia, a que los shocks de liquidez fuerzan a algunos agentes a retirar capital de las firmas de forma prematura, lo que genera un retorno de liquidación demasiado bajo, es decir, los mercados accionarios permitirían manejar dichos golpes de liquidez.

Por su parte, para Bencivenga y Smith (1991) las necesidades futuras de liquidez fuerzan a una acumulación de capital improductivo; en este escenario, la intermediación financiera provee de los fondos necesarios, haciendo de la mencionada acumulación algo innecesario. El modelo de King y Levine (1993) establece que un mejor sistema financiero mejora la probabilidad de éxito de la innovación, y por lo tanto acelera el crecimiento económico; además, el estudio involucra el ratio obligaciones líquidas del sistema financiero a PIB como una medida de su profundidad (la del sistema financiero).

Por su parte, en el modelo de Bencivenga et al. (1996) se enfatiza el rol del mercado de capitales como proveedor de liquidez para los agentes de largo plazo. Empíricamente, King et al. (1993) evidencian, mediante un análisis transversal de países, que el sistema financiero puede mejorar el crecimiento económico. No obstante, dicho estudio no utiliza de forma explícita ninguna variable concerniente al mercado accionario, sino que, al igual que King y 
Levine (1993), utiliza el ratio obligaciones líquidas del sistema financiero a PIB, es decir, un indicador de profundidad.

Levine (1997) aborda el tema tanto conceptual como empíricamente y encuentra una relación positiva entre desarrollo financiero y crecimiento económico. Finalmente, Bekaert y Harvey (1998) vinculan de forma explícita el crecimiento económico con los mercados de capitales, particularmente con los mercados accionarios. Si bien la mayor parte de su trabajo se limita al campo de lo teórico, el autor expone medidas de desarrollo del mercado accionario, a saber, market capitalization, value traded, market capitalization/GDP, value traded/GPD y Turnover, aunque, vale decir, el análisis cuantitativo no va más allá de una correlación entre las anteriores variables y el crecimiento económico, evidenciando una relación positiva.

Sin embargo, la utilización de Turnover, de ahora en adelante TO, Valued Traded/GDP, de ahora en adelante VT, y Market Capitalization/GDP, de ahora en adelante SC, se remonta a mucho antes de Bekaert y Harvey (1998). Su utilización explícita, como medida de desarrollo del mercado accionario, se la puede apreciar en el estudio de los mercados accionarios y su relación con las decisiones de financiamiento de las empresas, realizado por Demirguc-Kunt y Maksimovic (1995), aunque dichos autores dicen haberlas tomado de Pagano (1993). Pero Atje y Jovanovic (1993) también utilizan una de las variables en ese mismo año, a saber: $V T$; los autores encuentran que ésta variable tiene una relación positiva con el crecimiento económico.

A pesar de ello, no es sino con el trabajo de Demirguc-kunt y Levine (1996) que a nuestra terna de variables se las aborda de manera formal, definiéndolas y contextualizándolas. A partir de allí, la literatura las ha considerado como medidas predilectas para representar el desarrollo del mercado accionario. Por ejemplo, y si bien no en la misma línea sí en el mismo sentido de nuestra investigación, Demirguc-kunt y Levine (1996) encuentran que un TO elevado, es decir, un mercado accionario activo, sí que favorece un crecimiento de las firmas; sin embargo, desestima el aporte de SC. Por su parte Levine (1997) observa diferencias entre países ricos y pobres respecto a los indicadores del desarrollo de mercados accionarios. En concreto, observa que en los primeros existe una proporción mayor de Stock Market Trading, ${ }^{2}$ y del Stock Market Capitalization ${ }^{3}$ con respecto a los segundos; de lo anterior se podría inferir que TO eventualmente también sería mayor. A su vez Levine y Zervos (1998a) utiliza entre otras medidas, los ratios inicialmente mencio-

2 Lo cual influiría en VT.

3 Lo cual influiría en SC. 
nados para determinar si la liberación del control de capitales, es decir, la integración internacional, influye en el desarrollo del mercado accionario. Sus resultados sugieren que los mercados accionarios se vuelven más grandes y más líquidos, aunque también más volátiles, en la medida en la que se liberan los flujos de capitales.

Reafirmando el papel jugado por TO, VT y SC Levine y Zervos (1998b) definitivamente los contextualizan como medidas de liquidez a los dos primeros ratios, mientras que al tercero lo ubican como medida de tamaño del mercado accionario. De allí en adelante, e intendo responder a cómo influyen tanto los mercados accionarios como el sistema financiero en el desarrollo económico, se han desprendido un sin número de estudios, de los cuales mencionamos a Rousseau y Wachtel (2000), Arestis y Demetriades (2001), Beck y Levine (2004), Arena (2008), Cooray (2010), Wu et al. (2010), Cheng (2012), Carp (2012), Al Nasser (2015), Durusu-ciftci et al. (2017), Hou y Cheng (2017). En las investigaciones nombradas se utilizaron, sino a los tres, por lo menos a uno de los ratios TO, VT y/o SC.

\section{Metodología}

\subsection{Las Variables}

El crecimiento se midió a través de la variación del PIB per cápita, es decir, como $\operatorname{Ln}\left(y_{i, t}\right)$ - Ln $\left(y_{i, t-1}\right)$, en donde $y$ representa el PIB per cápita. Como variables de liquidez se tomaron las medidas expuestas en la sección anterior, las cuales, junto con SC, permiten capturar además el desarrollo del mercado accionario en su conjunto. Dichas medidas son:

- Size-Capitalization (SC): este mide el tamaño del mercado accionario con respecto a la economía total, y es igual al valor monetario total de las acciones listadas en la bolsa doméstica divido entre el PIB. Esta medida no es de liquidez, sino de desarrollo de mercado. En general, ésta medida captura la importancia del mercado accionario dentro de una economía, de ahí que se deba controlar por esta variable. Cabe esperar que en economías más desarrolladas, al menos en el plano financiero, su proporción respecto al PIB sea mayor.

- Liquidez (Lq): esta puede ser capturada a través del Turnover, y del Value Traded.

- Turn-Over (TO): es igual al valor monetario de las acciones tranzadas en la bolsa doméstica durante un período, dividido entre el valor monetario del total de acciones listadas en la bolsa doméstica en el 
mismo período. Esta medida intenta capturar el número de veces que las acciones son tranzadas (cambian de manos) en el respectivo mercado accionario durante un intervalo de tiempo. Mientras más elevado el ratio, más líquidas son son las acciones de dicho mercado.

- Value-Traded (VT): Medida igual al valor monetario del total de acciones tranzadas en la bolsa doméstica durante un período, dividido entre el PIB. A diferencia del SC, esta medida involucra solo las acciones tranzadas, es decir, captura la importancia, desde un contexto transaccional y de actividad de la bolsa, de las acciones de un mercado respecto a la economía.

De acuerdo con Demirgui-kunt y Levine (1996) y Levine y Zervos (1996), SC mide el grado en el cual el inversor puede diversificar el riesgo. Por su parte, un alto TO está asociado con bajos costos de transacción, mientras que el VT mide el volumen tranzado como una porción del output nacional, y por lo tanto refleja positivamente la liquidez de toda la economía. Sin embargo, y ya que los mercados financieros están mirando hacia el futuro, VT involucra un error potencial; si los mercados anticipan unas ganancias considerables, los precios de las acciones se elevarán hoy. Esto a su vez elevaría el valor de las transacciones y por lo tanto también el Value Traded, lo cual significaría que el mencionado ratio se eleva sin que exista un incremento en el número de transacciones o una reducción en sus costos (los de transacción). Este efecto contaminaría, además, la capitalización. Una forma de medir la influencia del efecto del precio es mirar el SC y el VT juntos; así, el efecto del precio influye en ambos indicadores, pero solo el VT está relacionado directamente con el trading. Por esta razón, se incluye el CS además del VT en la regresión que se expondrá posteriormente (Levine y Zervos, 1998b).

Con base en estudios previos, como los de Levine y Zervos (1998b); Arestis y Demetriades (2001); Beck y Levine (2004); Wu et al. (2010); Hou y Cheng (2017); Fufa y Kim (2018) es necesario controlar el desarrollo bancario (DB), el cual, en el presente estudio, será capturado a través del crédito bancario concedido al sector privado, como porcentaje del PIB. La tasa de natalidad $(T N)$ y el gasto público $(G P)$ también se consideran. Ya que se ha encontrado evidencia de que la apertura comercial $(A C)$ es un factor que influye en el crecimiento económico (Pradhan et al., 2014; Fetahi-vehapi et al., 2015), se considera también dicha medida. De este modo, $A C$ se obtiene a través del valor total del comercio de un país respecto a su PIB. Finalmente, y como exponen Billmeier y Massa (2009), la calidad de las instituciones de un país repercute en el desarrollo del mercado de acciones; además, la existencia de transparencia y regulaciones incrementan la confianza del inversor (Pagano, 1993), 
por lo tanto es necesario agregar a la corrupción como un factor de control, la que es capturada mediante el índice de percepción de la corrupción de transparencia internacional. No obstante, para evitar confusiones en la relación corrupción-crecimiento económico se denominará IT, significando que una puntuación alta en este índice es sinónimo de mayor transparencia por parte de un país, es decir, es menos corrupto. Mientras que una puntuación menor en IT significaría lo contrario. Finalmente, se controla por la inflación (INFL).

En resumen, la variable dependiente es el crecimiento per cápita del PIB (growth), mientras que las explicativas de interés son $S C$, TO y VT y las de control son $D B, T N, G P, A C$ e IT.

\subsection{El modelo}

Como ya se expuso, el impacto del desarrollo del mercado de capitales en el crecimiento económico ha sido materia de estudio por parte de un gran número de académicos; no obstante, es de especial interés para el presente artículo el realizado por Beck y Levine (2004). Estos autores utilizan datos de panel, estimados por el método de momentos generalizados (GMM). Siguiendo las directrices de los mencionados autores, y retomando a Arellano y Bond (1991) y Arellano y Bover (1995) la regresión se escribiría de acuerdo con la ecuación (1).

$$
y_{i, t}-y_{i, t-1}=\alpha y_{i, t-1}+\beta^{\prime} X_{i, t}+\eta_{i}+\varepsilon_{i, t}
$$

en donde y, como se mencionó anteriormente, es el logaritmo del PIB per cápita, $X$ representa el conjunto de variables explicativas, las cuales a parte del rezago del PIB per cápita, son tanto las variables de interés como las de control, $\eta$ es un efecto no observado específico del país, $\varepsilon$ es el término del error, y los subíndices i y t representan el país y el tiempo respectivamente. Por su parte, Arellano y Bond (1991) proponen las diferencias de la ecuación 1 (2),

$$
\left(y_{i, t}-y_{i, t-1}\right)-\left(y_{i, t-1}-y_{i, t-2}\right)=\alpha\left(y_{i, t-1} y_{i, t-2}\right)+\beta^{\prime}\left(X_{i, t}-X_{i, t-1}\right)+\left(\varepsilon_{i, t}-\varepsilon_{i, t-1}\right)
$$

A decir de Beck y Levine (2004), la ecuación 2 elimina el efecto específico del país; sin embargo, introducen un nuevo sesgo: dado que por construcción el nuevo término del error, $\varepsilon_{i, t}-\varepsilon_{i, t-1}$ está correlacionado con el rezago de la variable dependiente $y_{i, t-1}-y_{i, t-2}$. Bajo las suposiciones que (a) el término del error, $\varepsilon$, no está serialmente correlacionado, y (b) las variables explicativas, 
$X$, son débilmente exógenas, Arellano y Bond proponen las siguientes condiciones de momento.

$$
\begin{aligned}
& E\left[y_{i, t-s}\left(\varepsilon_{i, t}-\varepsilon_{i, t-1}\right)\right]=0 \text { Para } s \geq 2 ; t=3, \ldots, T, \\
& E\left[X_{i, t-s}\left(\varepsilon_{i, t}-\varepsilon_{i, t-1}\right)\right]=0 \text { Para } s \geq 2 ; t=3, \ldots, T,
\end{aligned}
$$

En resumen, Arellano y Bond (1991) proponen que la regresión de datos de panel sea estimada por GMM de dos pasos (two step), mientras Beck y Levine (2004) utilizan, además, el método GMM en diferencias. No obstante, también se estimará con un GMM one-step con robust VCE (Stata.com, 2018). Además, se estimaron variantes de los mencionados modelos, lo cual se describe más en detalle en la sección resultados. Por lo tanto, nuestro estudio utiliza datos de panel, estimados con el método GMM, bajo las consideraciones mencionadas.

\subsection{Datos}

Los datos necesarios para la elaboración de los índices fueron tomados de la World Federation of Exchanges (WFE) y del Banco Mundial. Por su parte el índice de percepción de la Corrupción se obtuvo de Transparencia Internacional. Los datos corresponden al período 2003-2017, esto debido a que para el caso de Latinoamérica los datos necesarios para la creación de índices no están disponibles sino a partir de 2003. ${ }^{4}$

En la tabla 1 se observa la estadística descriptiva. Lo primero que llama la atención es que no existe un número constante de observaciones; la muestra no es balanceada. De acuerdo con la mencionada tabla, durante el período analizado, el crecimiento medio del PIB per cápita fue de $6 \%$, el máximo crecimiento fue de $27 \%$, y el mínimo de $-31 \%$, es decir, decreció; por su parte, la desviación estándar del crecimiento rondó el 10\%.

Llama la atención que los valores mínimos y máximos sean del gigante sudamericano, esto sin duda es muestra de su inestabilidad económica. Con respecto a $S C$ se puede mencionar que en promedio el mercado accionario representa un $46 \%$ del PIB. Los valores mínimos y máximos de dicha medida corresponden respectivamente a Costa Rica (4,3\%), y Chile (156\%). Por otro lado, en promedio durante el período analizado se tranzó (o rotó, TO) un $18 \%$ del valor total de las acciones listadas; lo máximo fue $126 \%$ el valor

4 Esto en la página web de la WFE. 
de las acciones listadas; mientras que lo mínimo fue un 0,3\%, estos valores corresponden a Brasil y Barbados respectivamente.

Tabla 1. Estadística Descriptiva

\begin{tabular}{cccccc}
\hline Variable & Obs & Mean & Std. Dev. & Min & Max \\
\hline Growth & 148 & 0,0635 & 0,1001 & $-0,3180$ & 0,2751 \\
SC & 115 & 0,4665 & 0,3069 & 0,0430 & 1,5640 \\
TO & 119 & 0,1846 & 0,2183 & 0,0031 & 1,2674 \\
VT & 115 & 0,0915 & 0,1059 & 0,0007 & 0,4424 \\
TN & 154 & 16,9618 & 3,2583 & 9,4000 & 22,7850 \\
AC & 154 & 0,6609 & 0,3161 & 0,2211 & 1,6670 \\
IT & 148 & 5,1005 & 6,2007 & 2,5000 & 7,8000 \\
DB & 134 & 0,4728 & 0,2824 & 0,0968 & 1,1253 \\
GP & 154 & 0,1452 & 0,0288 & 0,0993 & 0,2020 \\
INFL & 163 & 5,6631 & 5,0761 & $-1,1128$ & 36,200 \\
\hline
\end{tabular}

Fuente: elaboración propia.

Por su parte, el valor tranzado (VT) en promedio representó el $9 \%$ del PIB, mientras que el valor mínimo fue prácticamente el $0 \%$ del PIB y el máximo fue de un $44 \%$ del PIB. Debido a que es una variable de interés, los créditos bancarios al sector privado en promedio representaron un $47 \%$ del PIB, lo que muestra el grado de importancia de la bancapara Latinoamérica: lo mínimo y máximo fueron, respectivamente un 9\% para Argentina en 2004 y un $112 \%$ para Chile en 2017. Similar análisis se obtiene de la estadística descriptiva para el resto de variables; sin embargo, debido a que no son de principal interés en el presente estudio, se prescinde de su descripción. Adicionalmente, en la tabla 2 constan las correlaciones entre las distintas variables.

Tabla 2. Correlaciones de las distintas variables

\begin{tabular}{|c|c|c|c|c|c|c|c|c|c|c|}
\hline & Growth & SC & $\mathrm{TO}$ & VT & $\mathrm{TN}$ & AC & IT & DB & GP & INFL \\
\hline Growth & 1 & & & & & & & & & \\
\hline SC & 0,1913 & 1 & & & & & & & & \\
\hline $\mathrm{TO}$ & $-0,1294$ & 0,0253 & 1 & & & & & & & \\
\hline VT & 0,0507 & 0,5009 & 0,8153 & 1 & & & & & & \\
\hline $\mathrm{TN}$ & 0,2095 & $-0,4277$ & $-0,251$ & $-0,4586$ & 1 & & & & & \\
\hline AC & $-0,037$ & 0,2194 & $-0,4189$ & $-0,3027$ & 0,1073 & 1 & & & & \\
\hline
\end{tabular}




\begin{tabular}{ccccccccccc}
\hline & Growth & SC & TO & VT & TN & AC & IT & DB & GP & INFL \\
\hline IT & $-0,1015$ & 0,5429 & 0,0204 & 0,2394 & $-0,4299$ & 0,2376 & 1 & & & \\
DB & $-0,1428$ & 0,6662 & 0,0969 & 0,3872 & $-0,7062$ & 0,3966 & 0,5809 & 1 & & \\
GP & $-0,2116$ & $-0,2201$ & 0,4478 & 0,3737 & $-0,5192$ & $-0,7309$ & $-0,149$ & $-0,0405$ & 1 & \\
INFL & $-0,143$ & $-0,379$ & 0,0056 & $-0,1434$ & $-0,0335$ & $-0,04326$ & $-0,2078$ & $-0,3438$ & 0,34 & 1 \\
\hline
\end{tabular}

Fuente: elaboración propia.

Como se puede observar, la mayor parte de las variables se correlacionan según lo que el sentido común sugeriría; por ejemplo, es razonable pensar que $S C$ y Growth vayan en el mismo sentido, del mismo modo que sucede con la correlación entre $V T$ y Growth. No obstante, llama la atención que TO se correlacione de forma negativa con respecto al crecimiento, lo mismo para el caso de $A C$; más curioso aún es que la transparencia IT correlacione de forma negativa con el crecimiento económico, toda vez que mientras mayor sea IT menos corrupto significa que es un país. La tabla 2 provee mayores detalles.

\section{Resultados}

Cabe mencionar que, de acuerdo con Beck y Levine (2004), se estimaron varios modelos. Dichos modelos son: (a) una regresión de Mínimos Cuadrados Ordinarios (MCO); (b) un modelo de control GMM Arellano-Bond de acuerdo con la ecuación 1, en donde solo consta $T O$ y $V T$, que son nuestras variables de interés, y el rezago del crecimiento; (c) un segundo modelo bajo el mismo método Arellano Bond del modelo de control, pero en este caso con todas las variables explicativas; (d) un tercer modelo, el cual es estimado con base en un Arellano Bond en diferencias, es decir la ecuación 2; (e) un cuarto modelo, el cual se estima con base en el modelo Arellano Bond en diferencias, es decir, ecuación 2, pero twostep; (f) un quinto modelo, que es el cuarto modelo pero estimado con vce (robust); (g) un sexto modelo, que es un Arellano Bond en diferencias estimado con Robust, pero en el que, en lugar de $S C$ en niveles, se utilizó su rezago; y (h) finalmente, un séptimo modelo, que es similar al Modelo 6, pero en lugar de $D B$ en nivel consta su rezago. Los resultados se presentan en la tabla 3.

Como ya se mencionó, las variables de interés en el presente artículo principalmente son TO y VT ya que, de acuerdo con Levine y Zervos (1998b), éstas representarían la liquidez; no obstante, también se puso atención a las variables $S C$ y $D B$, mientras que el resto serán comentadas brevemente de ser. Así, en el modelo MCO las variables de interés TO y $V T$, son significativas al $5 \%$ y $10 \%$ respectivamente; esto se corresponde con la literatura, aun 
cuando el signo de TO es diferente al esperado. Además, se observa que el gasto público influye de forma negativa en el crecimiento económico, lo cual per se no contradice la literatura. ${ }^{5}$ En el modelo de control, TO y VT siguen siendo significativas al 10\%, la misma significancia que tiene el rezago del crecimiento. En el modelo 2 TO deja de ser significativo si se lo compara con cualquiera de los dos modelos anteriores mientras el VT sigue siendo significativo. También cabe mencionar que la variable IT obtiene un resultado igualmente significativo; sin embargo, el resto de variables no lo son. Es decir, en el Modelo 2 y ya utilizando GMM, lo cual ayuda a controlar por endogeneidad, se pierde significancia en uno de los indicadores de interés, a saber TO. En el Modelo 3 se observan prácticamente los mismos resultados que en el modelo anterior, lo que da cuenta de la coherencia de los resultados del modelo previo; además, indicaría que los efectos fijos parecieran ser irrelevantes.

Tabla 3. Resultados distintos Modelos.

\begin{tabular}{|c|c|c|c|c|c|c|c|c|}
\hline Variable & $\mathrm{MCO}$ & Control & Modelo_2 & Modelo_3 & Modelo_4 & Modelo_5 & Modelo_6 & Modelo_7 \\
\hline SC & $-0,0526$ & & $-0,0572$ & $-0,0572$ & $-0,0042$ & $-0,0572$ & & \\
\hline TO & $-0,3926^{* *}$ & $-0,2497^{*}$ & $-0,1243$ & -01243 & 0,1031 & $-0,1242^{*}$ & $-0,1686^{* * *}$ & $-0,2036^{* * *}$ \\
\hline VT & $0,9481^{*}$ & $0,9163^{*}$ & $1,1396^{*}$ & $1,1396^{*}$ & 0,0000 & $1,1396^{* *}$ & 0,1282 & 0,119 \\
\hline $\mathrm{TN}$ & $-0,0067$ & & 0,017 & 0,017 & 0,3866 & 0,017 & 0,0272 & 0,0196 \\
\hline $\mathrm{AC}$ & $-0,2391$ & & $-0,3241$ & $-0,3241$ & $1,9626^{*}$ & $-0,3241$ & $-0,7249^{*}$ & $-0,7383^{*}$ \\
\hline IT & $-0,0123$ & & $-0,0257^{*}$ & $-0,0257^{*}$ & 0,1279 & $-0,0257^{* * *}$ & $-0,0171^{* *}$ & $-0,0159^{* *}$ \\
\hline DB & $-0,0973$ & & $-0,1819$ & $-0,1819$ & 6,8101 & $-0,1819$ & $-0,0917$ & \\
\hline GP & $-2,1525^{*}$ & & $-3,4278$ & $-3,4278$ & 0,0000 & $-3,4278^{*}$ & $-3,0777$ & $-3,5387$ \\
\hline INFL & $-0,0037$ & & $-0,0029$ & $-0,0029$ & $-0,0106$ & $-0,0029$ & $-0,0033$ & $-0,0031$ \\
\hline \multicolumn{9}{|l|}{ growth } \\
\hline L1. & & $0,2224^{*}$ & $-0,1043$ & $-0,1043$ & $-3,1037^{*}$ & $-0,1043$ & $-0,2117^{* *}$ & $-0,1962^{*}$ \\
\hline \multicolumn{9}{|l|}{$\ln$ GDP } \\
\hline L1. & & & $-0,0789$ & $-0,0789$ & $-1,2378$ & $-0,0789$ & $-0,1305$ & $-0,1495$ \\
\hline \multicolumn{9}{|l|}{ SC } \\
\hline L1. & & & & & & & $0,4545^{* * *}$ & $0,4676^{* * *}$ \\
\hline
\end{tabular}

Continúa

5 Ya que en la literatura existen estudios que respaldan las posiciones contrapuestas. Es decir hay respaldo respecto a una influencia positiva en el crecimiento económico, pero también lo hay para el caso de una influencia negativa (Bose, 2007). 


\begin{tabular}{|c|c|c|c|c|c|c|c|c|}
\hline Variable & $\mathrm{MCO}$ & Control & Modelo_2 & Modelo_3 & Modelo_4 & Modelo_5 & Modelo_6 & Modelo_7 \\
\hline L1. & & & & & & & & 0,0124 \\
\hline cons & 0,7435 & 0,0056 & 1,2881 & 1,2881 & 0 & 1,2881 & 1,521 & 1,8407 \\
\hline $\begin{array}{c}\text { Sargan } \\
\text { Test }\end{array}$ & . & 0,2973 & 0,5657 & 0,5657 & . & . & . & . \\
\hline
\end{tabular}

* Connota significancia al $10 \%$.

** Connota significancia el $5 \%$.

*** Connota significancia al $1 \%$ respectivamente.

Fuente: elaboración propia.

Por su parte, en el modelo 4, el cual es un Arellano Bond en diferencias twostep, se observa que se pierde la poca significancia que tenían las variables $V T$ e IT. No obstante, $A C$ y el rezago del crecimiento ahora son significativos al $10 \%$. Esta situación se revierte en el modelo 5, en donde nuestras 2 variables de interés, TO y VT son significativas al $10 \%$ y $5 \%$, respectivamente; además, se suman a éstas las variables IT y GP. Los resultados anteriores son interesantes, toda vez que se comprueba que, efectivamente, la liquidez influye en el crecimiento económico; sin embargo, y como se observa a lo largo de los distintos modelos, TO muestra un signo negativo, lo cual sería contra intuitivo. Por su parte, y en un intento por saber si tanto $S C$ como $D B$ explican de alguna forma el crecimiento económico, se procedió a estimar los modelos 6 y 7; en estos modelos se observa que TO sigue siendo significativo, pero, aún más llamativo, es el rezago de $S C$, que es significativo al $1 \%$, aun cuando se ha perdido significancia en la variable $V T$.

Además, hay que mencionar que $D B$ no es significativo tampoco en su rezago. Los resultados de los modelos 5 al 7 sugieren que, en efecto, la consideración de que existe hetorecedasticidad es correcta. Estimando de forma robusta la significancia de algunos de los coeficientes, esta mejora, como sucede, por ejemplo con el TO; sin embargo, la de VT se pierde en los modelos 6 y 7. Por otra parte, y debido a que la muestra no es muy grande, la interpretación de las estimaciones con robust debe ser cautelosa; considerando lo anterior, y ya controlando el modelo por heterocedasticidad, los resultados sugieren que la rotación accionario síinfluye en el crecimiento económico. Cabe mencionar que SC, en su rezago, también lo hace, es decir, en un contexto general, el desarrollo accionario visto a través de sus distintas medidas influye en el crecimiento económico.

Adicionalmente, los signos de nuestras variables de interés se mantienen en todos los modelos, incluso en el de control; la significancia por su parte no es constante, aunque se obtuvieron algunas de hasta el $1 \%$. A nivel general, 
se podría mencionar que la Capitalización (SC) de un mercado accionario explica el crecimiento económico del año subsiguiente; a su vez, la proporción del volumen tranzado (VT) respecto al PIB, así como la rotación del mercado accionario (TO), también explican el crecimiento económico. A nuestro criterio, la estimación más adecuada se obtiene con base en el modelo Arellano Bond en diferencias estimado con Robust, en el cual consta el rezago de SC. A su vez $D B$ en ninguno de los modelos resulta significativo, ni siquiera al $10 \%{ }^{6}$ También se observa que al estimar con GMM el signo del coeficiente de TN cambia con respecto a los valores obtenidos con $\mathrm{MCO}$. Cabe mencionar que TO presenta un signo negativo, esto sin duda llama la atención debido a la clara contraposición con lo mencionado por Levine y Zervos (1998b).

Por otro lado, y debido a que Barbados, Bermudas y Jamaica podrían ser considerados como atípicos (outliers) dentro de la muestra, o que no forman parte de Latinoamérica en sentido estricto, el estudio estimó los mismos modelos ya descritos, pero prescindiendo de los mencionados países.

En la tabla 4 se observa que, en general, los resultados no varían sustancialmente cuando se estiman las variables teniendo en cuenta a Barbados, Bermuda y Jamaica. Lo que se observan son leves variaciones de significancia en algunos de los coeficientes, pero el sentido es prácticamente el mismo. Cabe mencionar que, por ejemplo, el signo TO se mantiene en ambos escenarios, es decir, para este caso los posibles outliers no son los que provocan un resultado contra-intuitivo respecto a la influencia que tiene la rotación accionaria para con el crecimiento económico.

Tabla 4. Resultados sin Barbados, Bermuda y Jamaica

\begin{tabular}{ccccccccc}
\hline Variable & MCO & Control & Modelo_2 & Modelo_3 & Modelo_4 & Modelo_5 & Modelo_6 & Modelo_7 \\
\hline SC & 0,0070 & & $-0,0143$ & $-0,0143$ & 0,3058 & $-0,0143$ & & \\
TO & $-0,3454^{*}$ & $-0,2934^{*}$ & $-0,0577$ & $-0,0577$ & 0,0000 & $-0,0577$ & $-0,1432^{*}$ & $-0,1696^{* * *}$ \\
VT & $0,8916^{*}$ & $0,9998^{*}$ & $1,1385^{*}$ & $1,1385^{*}$ & 0,0000 & $1,1385^{* * *}$ & $0,3413^{* *}$ & $0,3348^{*}$ \\
TN & $-0,0029$ & 0,0092 & 0,0092 & 0,3335 & 0,0092 & $-0,0019$ & $-0,0047$ \\
AC & $-0,0004$ & $-0,0047$ & $-0,0047$ & $0,0082^{*}$ & $-0,0047$ & $-0,0085^{* *}$ & $-0,0089^{* * *}$ \\
IT & $-0,0083$ & $-0,0266^{* *}$ & $-0,0266^{* *}$ & 0,0307 & $-0,0266^{* * *}$ & $-0,0189^{*}$ & $-0,0192^{* * *}$ \\
DB & $-0,1446$ & $-0,0495$ & $-0,0495$ & 8,0297 & $-0,0495$ & 0,0705 & \\
GP & $-1,0301$ & $-1,7446$ & $-1,7446$ & 0,0000 & $-1,7446$ & $-1,2291$ & $-1,7285$ \\
& & & & & & & Continúa
\end{tabular}

6 No obstante es posible que DB impacte en el crecimiento económico solo a partir de cierto umbral. Véase Ruiz (2018). 


\begin{tabular}{|c|c|c|c|c|c|c|c|c|}
\hline Variable & $\mathrm{MCO}$ & Control & Modelo_2 & Modelo_3 & Modelo_4 & Modelo_5 & Modelo_6 & Modelo_7 \\
\hline \multicolumn{9}{|l|}{ growth } \\
\hline L1. & & 0,2119 & $-0,0364$ & $-0,0364$ & $-0,4212$ & $-0,0364$ & $-0,0951$ & $-0,0869$ \\
\hline INFL & & & $-0,0031$ & $-0,0032$ & $-0,0029$ & $-0,0032$ & $-0,0033$ & $-0,0037$ \\
\hline \multicolumn{9}{|l|}{ lnpib } \\
\hline L1. & & & $-0,1559$ & $-0,1559$ & $-1,1321$ & $-0,1559$ & $-0,2455$ & $-0,2712^{*}$ \\
\hline \multicolumn{9}{|l|}{ SC } \\
\hline L1. & & & & & & & $0,4417^{* * *}$ & $0,4655^{* * *}$ \\
\hline \multicolumn{9}{|l|}{ DB } \\
\hline L1. & & & & & & & & 0,1890 \\
\hline _cons & 0,3625 & 0,0048 & 1,8462 & 1,8462 & 0,0000 & 1,8462 & 2,7564 & $3,0742499 *$ \\
\hline
\end{tabular}

Los autores consideran que si bien en un sentido estricto Barbados, Bermudas y Jamaica no forman parte de Latinoamérica, sino de América Latina y el Caribe, dados los resultados símiles entre la tabla 3 y 4 , y ya que el contar con dichos países se agrega generalidad al estudio, se opta por establecer la discusión y las conclusiones con base en la tabla 3.

\section{Discusión}

Los resultados obtenidos en el presente estudio plantean directrices para los creadores de políticas. Por un lado, que el rezago de SC contribuya al crecimiento económico hace sentido, toda vez que se asume que un incremento de la capitalización es una revalorización de las acciones o un aumento de capital, los cuales la mayoría de las veces se dan ante expectativas favorables para la empresa, ya sea a nivel de ingresos o de emprendimiento de proyectos. Para el caso de los proyectos, si bien se espera sean emprendidos lo antes posible, sus efectos se verán más allá del corto plazo, es decir, una vez sean culminados y pasen a formar parte de la producción de la empresa. Es razonable pensar que el tiempo para la culminación de un proyecto a nivel corporativo sea, cuando menos, un año. En este sentido, la economía en general sentirá los efectos de nuevos proyectos, en el año siguiente al incremento de capital de las empresas, como mínimo. Por su lado, el hecho de que el nivel de rotación dentro de una bolsa tenga un efecto negativo en el crecimiento 
económico resulta contraintuitivo; significaría que mientras más veces una acción cambia de manos a la economía le va mal. Una explicación a ello vendría de la mano de movimientos especulativos y pánicos, lo cual provocaría un desempeño económico malo; los inversores desearán deshacerse de sus acciones, promoviendo una alta rotación. No obstante, lo anterior debería verse a la luz de Amihud (1989); además, no es más que una suposición y los aspectos de trasfondo quedan pendientes. Finalmente, hay que mencionar que en la medida en que existe un mayor valor tranzado se ve un crecimiento económico que está en línea con lo esperado. Un mayor valor tranzado podría significar que las acciones incrementaron su precio, lo que en general motiva a una visión positiva de la economía; esto también podría significar que las empresas están obteniendo rentabilidades muy buenas, con lo cual el inversionista valora más las acciones. De trasfondo, podría encontrarse un mayor consumo por parte de los ciudadanos, lo que al final del día influye en el crecimiento económico.

Los resultados son coherentes con los esfuerzos que se llevaron a cabo por parte de los distintos países latinoamericanos. Así, durante los años noventa gran parte de Latinoamérica implementó políticas liberales, intentando dinamizar los mercados de capitales, ampliar el número de empresas listadas en bolsa y facilitar la entrada de inversores. Dichas políticas se reflejan, por ejemplo, en la capitalización (SC), la cual, para nuestro caso, impacta de forma positiva en el crecimiento económico, es decir, aquellas políticas resultaron ser relativamente efectivas y lograron incrementar la capitalización de los diferentes mercados accionarios; en otras palabras, las empresas obtuvieron mayores recursos para sus proyectos. En adición a esto, el efecto positivo del Valor Tranzado (VT), implícitamente transmite que los inversores confían en el mercado, toda vez que mayores valores tranzados implican perspectivas favorables respecto a la economía de un país. VT podría deberse además a una revalorización general de las acciones, atrayendo aún más inversionistas y facilitando el acceso a recursos por parte de las empresas. En el caso de TO su signo está en total contraposición a lo mencionado por la literatura. Los resultados sugerirían que para el caso de Latinoamérica la rotación de acciones tiene su mayor pico en momentos en los cuales la economía atraviesa dificultades; Además, deja traslucir movimientos especulativos y pánicos por parte de los inversores ante perspectivas económicas desfavorables, las cuales en efecto parecieran materializarse, de allí el signo negativo de TO.

De acuerdo con lo expuesto, la liquidez tiene un impacto ambiguo en el crecimiento económico a nivel latinoamericano; de todas formas, ya sea para bien o para mal, dicho impacto estaría por debajo de sus potencialidades 
(De la Torre et al., 2007), es decir, la liquidez debería y podría tener un mayor impacto en el crecimiento.

\section{Conclusiones}

El estudio presenta evidencia empírica de que, a nivel latinoamericano, la teoría se comprueba parcialmente. Por un lado, las medidas de liquidez efectivamente influyen en el crecimiento económico; sin embargo, para el presente caso, TO influye negativamente, lo cuál sin duda no nos deja indiferentes. Este resultado se mantiene desde las correlaciones, lo que llama la atención debido a la abundante literatura que existe respecto a que TO debería influir en forma positiva. Sin embargo, este resultado podría tener relación con las razones dadas anteriormente (Bencivenga et al., 1996) respecto a los problemas causados por la liquidez, aunque un análisis más profundo debería ser desarrollado en futuros estudios. Adicionalmente, se puede mencionar que $D B$ en ningún caso explica de forma significativa el crecimiento económico, ni aún su rezago, aunque se observa un cambio de signo cuando este se utiliza. También es interesante el hecho de que IT tenga una relación negativa con el crecimiento económico. No hay que olvidar que un IT alto denota un país menos corrupto, mientras un IT bajo denota un país más corrupto. Normalmente uno esperaría que un país con menor corrupción creciera más, por lo menos en el borde; esto no se observa en el presente estudio. Sin embargo, contextualizando el análisis, hay que considerar que Latinoamérica se ha caracterizado por una débil lucha contra la corrupción; más o menos por esta línea podría uno encontrar una explicación a los resultados.

Es necesario destacar que las estimaciones de los modelos al considerar a todos los países inicialmente mencionados son prácticamente iguales que al estimar sin considerar a Barbados, Bermuda y Jamaica. Es decir, en afán de obtener mayor generalidad de los resultados, lo más conveniente es apreciar la tabla 3; en otras palabras: los outliers no parecen afectar los resultados generales.

Como penúltimo punto, se refuerza el aporte del artículo en dos vías; el primero: sí, la liquidez medida como VT influye en el crecimiento económico; y sí, la liquidez medida como TO también lo hace, pero no en el sentido esperado. Finalmente, este estudio pretende no solo constituir un avance académico, sino además es un intento por proveer de herramientas al policymaker para que, sino correctas, tome decisiones cuando menos fundamentadas. 


\section{Referencias}

Aali-Bujari, A., Venegas-Martínez, F., \& Pérez-Lechuga, G. (2017). Impact of the stock market capitalization and the banking spread in growth and development in Latin American: A panel data estimation with System GMM. Contaduría y Administración, 62(5),1427-1441.

Al Nasser, O. M. (2015). Stock markets, banks, and economic growth: evidence from Latin American countries. International Journal of Economics and Finance, 7(2), 100-112.

Amihud, Y., Mendelson, H., \& Wood, R. (1989) R. Liquidity and the 1987 stock market crash. The Journal of Portfolio Management, 16, 65-69.

Arellano, M., \& Bond, S. (1991). Some tests of specification for panel data: Monte Carlo evidence and an application to employment equations. The Review of Economic Studies, 58(2), 277-299.

Arellano, M., \& Bover, O. (1995). Another look at the instrumental variable estimation of error-components models. Journal of Econometrics, 68(August 1990), 29-51.

Arena, M. (2008). Does insurance market activity promote economic growth? A cross-country study for industrialized and developing countries. Journal of Risk and Insurance, 75(4), 921-946.

Arestis, P., \& Demetriades, P. O. (2001). Financial development and economic growth: The role of stock markets. Journal of Money, Credit and Banking, $33(1), 16-41$.

Atje, R., \& Jovanovic, B. (1993). Stock markets and development. European Economic Review, 37, 632-640.

Beck, T., \& Levine, R. (2004). Stock markets, banks, and growth: Panel evidence. Journal of Banking \& Finance, 28, 423-442.

Bekaert, G., \& Harvey, C. R. (1998). Capital markets: An engine for economic growth. The Brown Journal of World Affairs, 5(1):33-53.

Bencivenga, V. R., \& Smith, B. D. (1991). Financial intermediation and endogenous growth. Review of Economic Studies, 58:195-209.

Bencivenga, V. R., Smith, B. D., \& Starr, R. M. (1996). Equity markets, transaction costs, and capital accumulation. An illustration. The World Bank Economic Review, 10(May), 241-265.

Billmeier, A., \& Massa, I. (2009). What drives stock market development in emerging markets, institutions, remittances, or natural resources? Emerging Markets Review, 10(1), 23-35.

Bose, N., Haque, E., \& Osborn, D. (2007) Public expenditure and economic growth: A disaggregated analysis for developing countries. The Manchester School, 75(5), 533-566. 
Carp, L. (2012). Can Stock Market Development Boost Economic Growth? Empirical Evidence from Emerging Markets in Central and Eastern Europe. Procedia Economics and Finance, 3(12), 438-444.

Cheng, S.-y. (2012). Substitution or complementary effects between banking and stock markets: Evidence from financial openness in Taiwan. Journal of International Financial Markets, 22(3), 508-520.

Cooray, A. (2010). Do stock markets lead to economic growth? Journal of Policy Modeling, 32(4), 448-460.

De la Torre, A., Gozzi, J.C., \& Schmukler, S. (2007). Capital Market Development: Wither Latin America? World Bank Policy Research Working Paper.

Demirguc-kunt, A., \& Levine, R. (1996). Stock market development and financial intermediaries: Stylized facts. World Bank Economic Review, 10(2), 291-321.

Demirguc-Kunt, A., \& Maksimovic, V. (1995). Stock Market Development and Firm Financing Choices. Washington D.C.: The world Bank.

Demirgui-kunt, A., \& Levine, R. (1996). Stock markets, corporate finance, and economic growth. The World Bank Economic Review, 10(2), 223-239.

Durusu-ciftci, D., Ispir, M. S., \& Yetkiner, H. (2017). Financial development and economic growth: Some theory and more evidence. Journal of Policy Modeling, 39(2), 290-306.

Fetahi-vehapi, M., Sadiku, L., \& Petkovski, M. (2015). Empirical analysis of the effects of trade openness on economic growth: An evidence for south east european countries. Procedia Economics and Finance, 19(15):17-26.

Francis, B. B., Hasan, I., \& Ofori, E. (2015). Investor protections, capital markets, and economic growth: the african experience. International Corporate Governance, 18, 239-272.

Fufa, T, \& Kim, J. (2018). Stock markets, banks, and economic growth: Evidence from more homogeneous panels. Research in International Business and Finance, 44(July2017), 504-517.

Goldsmith, R. W. (1969). Financial Structure and Development. New Haven: Yale University Press.

Harris, R. D. F. (1997). Stock markets and development: A re-assessment. European Economic Review, 41,139-149.

Hou, H. \& Cheng, S.-y. (2017). The dynamic effects of banking, life insurance, and stock markets on economic growth. Japan \& The World Economy, 41, 87-98.

KcKinnon, R. I. (1973). Money and capital in economic development. Washington DC: Brookins Institution.

King, R. G., \& Levine, R. (1993). Finance, entrepreneurship, theory and evidence, Journal of Monetary Economics, 32, 513-542. 
King, R. G., Levine, R., Quarterly, T., Aug, N., King, G., \& Levine, R. (1993). Finance and growth: Schumpeter might be right. The Quaterly Journal of Economics, 108(3), 717-737.

Levine, R. (1991). Stock markets, growth, and tax policy. The Journal of Finance, 46(4), 1445-1465.

Levine, R. (1997). Financial development and economic growth: Views and agenda. Journal of Economic Literature, 35(2), 688-726.

Levine, R., \& Zervos, S. (1996). Capital control liberalization and stock market development. Washington D.C.: The World Bank.

Levine, R., \& Zervos, S. (1998a). Capital control liberalization and stock market development. World Development, 26(7), 1169-1183.

Levine, R., \& Zervos, S. (1998b). Stock markets, banks, and economic growth. 88(3):537-558.

Lloyd, B. (1977). The role of capital markets in developing countries. Interconomics, 374(3), 92-106.

Lu, S.-s. (2013). The role of capital market efficiency in long-term growth: A quantitative exploration. Journal of Macroeconomics, 36, 161-174.

Lu, S.-s. (2013). The role of capital market efficiency in long-term growth: A quantitative exploration. Journal of Macroeconomics, 36, 161-174.

Pagano, M. (1993). The flotation of companies on the stock market. A coordination failure model. European Journal of Information Systems, 37, 1101-1125.

Pradhan, R. P., Arvin, M. B., Hall, J. H., \& Bahmani, S. (2014). Causal nexus between economic growth, banking sector development, stock market development, and other macroeconomic variables: The case of ASEAN countries. Review of Financial Economics, 23(4),155-173.

Rousseau, P. L. and Wachtel, P. (2000). Equity markets and growth: Crosscountry evidence on timing and outcomes, 1980-1995. Journal of financial banking, 24, 1933-1957.

Ruiz, J. L. (2018). Financial development, institutional investors, and economic growth. International Review of Economics and Finance, 54(August 2017), 218-224.

Schumpeter, J. A. (1911). The of economic development. Cambridge: Harvard University Press.

Shaw, E. S. (1973). Financial deepening in economic development. Nueva York: Oxford University Press.

Stata.com (2018). Manual for xtabond - Arellano-Bond linear dynamic paneldata estimation. 07/07/2018, de Stata. Recuperado de https:/ / www. stata.com/manuals13/xtxtabond.pdf 
Terceño, A., \& Guercio, B. (2011). El crecimiento economico y el desarrollo del sistema financiero. un analisis comparativo. Investigaciones Europeas de Dirección y Economía de la Empresa, 17, 33-46.

Wu, J.-l., Hou, H., \& Cheng, S. (2010). The dynamic impacts of financial institutions on economic growth: Evidence from the European Union. Journal of Macroeconomics, 32(3):879-891. 